\title{
Overexpression of microRNA-210 promotes chondrocyte proliferation and extracellular matrix deposition by targeting HIF-3 $\alpha$ in osteoarthritis
}

\author{
ZHIFU LI $^{1 *}$, DONGDONG MENG ${ }^{2 *}$, GUANGHENG LI ${ }^{1}$, JIANZHONG XU $^{1}$, KE TIAN $^{1}$ and YU LI ${ }^{1}$ \\ Departments of ${ }^{1}$ Orthopedic Surgery and ${ }^{2}$ Endocrinology, The First Affiliated Hospital of Zhengzhou University, \\ Zhengzhou, Henan 450052, P.R. China
}

Received February 11, 2015; Accepted December 1, 2015

DOI: $10.3892 / \mathrm{mmr} .2016 .4878$

\begin{abstract}
The present study aimed to determine the effect of microRNA (miR)-210 on osteoarthritis (OA). The expression levels of miR-210, type I and X collagen (COL1A1 and COL10A1) and matrix metallopeptidase 13 (MMP13) in OA and normal chondrocytes were determined using reverse transcription-quantitative polymerase chain reaction analysis. The OA chondrocytes were transfected with an miRNA precursor for miR-210 or a negative control. After $3,7,14$ and 21 days, the expression levels of miR-210 were examined, the proliferation of the OA chondrocytes were determined using an XTT assay and the protein levels of Ki67 and HIF-3 $\alpha$ were analyzed by Western blotting. After 21 days, the mRNA and protein levels of COL1A1, COL10A1 and MMP13 were analyzed. Th present study demonstrated that the expression levels of miR-210 and COL1A1 were lower, and the expression levels of COL10A1 and MMP13 were higher in the OA chondrocytes, compared with the levels of expression in the normal chondrocytes. Overexpression of miR-210 significantly promoted the proliferation of OA chondrocytes and induced the protein expression of Ki67. In addition, miR-210 overexpression markedly increased the expression of COL1A1 expression, but decreased the expression levels of COL10A1 and MMP13. A luciferase reporter assay confirmed the direct interaction between miR-210 and hypoxia-inducible factor (HIF)-3 $\alpha$. miR-210 did not alter the mRNA expression of HIF-3 $\alpha$, however, it suppressed the protein expression of HIF-3 $\alpha$. Additionally, HIF-3 $\alpha$ knockdown significantly promoted $\mathrm{OA}$ chondrocyte proliferation and increased the
\end{abstract}

Correspondence to: Dr Zhifu Li, Department of Orthopedic Surgery, The First Affiliated Hospital of Zhengzhou University, 1 Jianshe East Road, Zhengzhou, Henan 450052, P.R. China

E-mail: zhifulifm@163.com

*Contributed equally

Key words: microRNA-210, proliferation, hypoxia-inducible factor- $3 \alpha$, osteoarthritis
mRNA levels of COL1A1, whereas it decreased the mRNA levels of COL10A1 and MMP13. The results of the present study suggested that miR-210 may be a negative regulator of the progression of OA, which increases chondrocyte proliferation and prompts extracellular matrix deposition by directly targeting HIF-3 $\alpha$.

\section{Introduction}

Osteoarthritis (OA) is a common degenerative joint disease, which is characterized by joint tissue alterations, including articular subchondral bone changes, low-grade synovitis and cartilage loss. Patients with OA often endure joint pain, tenderness, stiffness and mobility difficulties $(1,2)$. Degradation of articular cartilage and extracellular matrix (ECM) is one of the predominant causes of OA (3). Chondrocytes are the only cells to form cartilage, and are responsible for ECM synthesis and deposition (4).

MicroRNAs (miRNAs) are 21 -nucleotide-long single-stranded RNAs, which regulate protein expression by interacting with the 3'-untranslated region (3'-UTR) of mRNA $(5,6)$. miRNAs have been reported to be involved in chondrogenesis and OA (7). For example, miR-21 controls the development of osteoarthritis by targeting growth differentiation factor 5 in chondrocytes (8). miRNA therapy using miR-146a and the miR-183 cluster may be an efficient therapeutic strategy for OA to alleviate joint pain and promote joint cartilage regeneration (9). miR-488 regulates the zinc transporter, SLC39A8/ZIP8, and reduces cartilage degradation during the pathogenesis of osteoarthritis (10). Overexpression of miR-148a promotes cartilage production and inhibits cartilage degradation by OA chondrocytes (11). miR-127-5p regulates the expression of matrix metalloproteinase 13 (MMP13) and interleukin-1 $\beta$-induced catabolic effects in human chondrocytes (12).

Of note, miR-210 can be induced by hypoxia in a wide range of normal and transformed cells (13), representing a major hypoxia-inducible miRNA (13). It has been demonstrated that miR-210 promotes proliferation in idiopathic pulmonary fibrosis fibroblasts (14) and ovarian cancer cells (15) in response to hypoxia. Chondrocyte metabolism operates under hypoxic conditions within the cartilage matrix (1), and 
the downregulation of miR-210 by hypoxia-inducible factor (HIF)-1 $\alpha$ decreases viability and induces apoptosis in hypoxic chondrocytes (16). However, the exact effects and underlying mechanism of miR-210 in OA chondrocytes remain to be fully elucidated.

The present study aimed to investigate the expression levels of miR-210 in OA chondrocytes, and to elucidate the role of miR-210 in the pathological process of OA. The present study has novel insight into the function of miR-210, which has the potential to improve treatment of cartilage disorders.

\section{Materials and methods}

Clinical specimens. Tissue specimens from knee joint cartilage were collected from five male patients and four female patients diagnosed with OA (mean age, 67 years; range, 61-73 years) who had undergone total knee arthroplasty at the First Affiliated Hospital of Zhengzhou University (Zhengzhou, China) between 2011 and 2014, and from six male and three female post-mortem donors (mean age, 62 years; range, 55-75 years) without OA. The study was approved by the Medical Ethics Committee of the First Affiliated Hospital of Zhengzhou University (Zhengzhou, China). Written prior informed consent was obtained from all patients. The specimens were immediately preserved in liquid nitrogen for subsequent experiments.

Cell isolation and cell culture. For cell isolation, articular cartilage was minced and digested in $0.15 \%$ (w/v) collagenase (CLS-2; Worthington, Lakewood, NJ, USA) in Dulbecco's modified Eagle's medium (DMEM; Gibco; Thermo Fisher Scientific, Inc., Waltham, MA, USA) supplemented with $10 \%$ fetal bovine serum (FBS; GE Healthcare Life Sciences, Logan, UT, USA), 100 U/ml penicillin (Gibco; Thermo Fisher Scientific, Inc.) and $100 \mathrm{mg} / \mathrm{ml}$ streptomycin (Gibco; Thermo Fisher Scientific, Inc.) for $16 \mathrm{~h}$ at $37^{\circ} \mathrm{C}$. The cells were filtered through a $100-\mathrm{mm}$ cell strainer (BD Biosciences, San Diego, CA, USA) and washed with sterile saline prior to culture or miRNA/mRNA isolation. Cells were cultured at a density of $5 \times 10^{4}$ cell/well, at $37^{\circ} \mathrm{C}$ and an atmosphere of $5 \% \mathrm{CO}_{2}$.

Reverse transcription-quantitative polymerase chain reaction (RT-qPCR) analysis. Total RNA was isolated from the tissues or cultured cells using TRIzol reagent (Invitrogen; Thermo Fisher Scientific, Inc.) and treated with 2 U of DNase I (Takara Biotechnology Co., Ltd., Dalian, China). The total RNA was reverse transcribed into cDNA using an Omniscript RT kit (Qiagen, Hilden, Germany) and random primers using the following temperatures: $42^{\circ} \mathrm{C}$ for $15 \mathrm{mins}, 85^{\circ} \mathrm{C}$ for $5 \mathrm{sec}$ and then incubation at $4^{\circ} \mathrm{C}$. The expression of miR-210 was determined using an miRNA qPCR detection kit (GeneCopoeia, Rockville, MD, USA) and the expression levels of COL2A1, COL10A1, MMP13 and HIF-3 $\alpha$ were determined using an SYBR Premix Ex Taq II kit (Takara Biotechnology Co., Ltd.) and gene-specific primers.(Table I) qPCR was performed in a Rotor-Gene RG-3000 Real-Time Thermal Cycler (Corbett Research, Sydney, Australia). The thermocycler conditions used were $94^{\circ} \mathrm{C} 2 \mathrm{~min}$ for initial denaturation, $94^{\circ} \mathrm{C}$ for $30 \mathrm{sec}$, $60^{\circ} \mathrm{C}$ for $15 \mathrm{sec}$, and $72^{\circ} \mathrm{C}$ for $15 \mathrm{sec} ; 2 \mathrm{sec}$ for plate reading for 40 cycles; and melt curve from 65 to $95^{\circ} \mathrm{C}$ The relative mRNA
Table I. Primers sequences for RT-qPCR.

\begin{tabular}{ll}
\hline Gene symbol & \multicolumn{1}{c}{ Sequence 5'-3' } \\
\hline miR-210 & F: ACACTCCAGCTGGGAGCCCCTG \\
& CCCACCGC \\
& R: TGGTGTCGTGGAGTCG \\
FOL2A1 & R: TGGTGGAGCAGCAAGAGC \\
COL10A1 & F: GATGAATACACCAAAGGCTAC \\
RMP-13 & R: GACTCGGCATTGGGAAGC \\
FIF-3 $\alpha$ & R: AACAGCTCCGCATCAACC \\
F6 & R: GCGGTCAGCAAGAGCATC \\
U6 & F: CTCGCTTCGGCAGCACA \\
& R: AACGCTTCACGAATTTGCGT \\
& F: GGGTCAGAAGGATTCCTATGTG' \\
& R: GTCCCAGTTGGTGACGATGC
\end{tabular}

F, forward; R, reverse; miR, microRNA; COL2A1, type II collagen; COL10A1, type X collagen; MMP-13, matrix metalloproteinase-13; HIF, hypoxia-inducible factor; RT-qPCR, reverse transcription quantitative polymerase chain reaction.

expression levels of miR-34 $\alpha$ and Atg4B were normalized using the $2^{-\Delta \Delta \mathrm{Cq}}$ method (17), relative to U6 small nuclear (sn) RNA and $\beta$-actin, respectively. The experiment was performed three times.

Transfection with mature miRNA and small interfering (si) $R N A$. The isolated OA chondrocytes were cultured in DMEM supplemented with $10 \% \mathrm{FBS}, 100 \mathrm{U} / \mathrm{ml}$ penicillin, $100 \mathrm{mg} / \mathrm{ml}$ streptomycin and $10 \mathrm{ng} / \mathrm{ml}$ basic fibroblast growth factor (bFGF; $\mathrm{R} \& \mathrm{D}$, Minneapolis, $\mathrm{MN}$, USA), at $37^{\circ} \mathrm{C}$ in a $5 \% \mathrm{CO}_{2}$ atmosphere. At passage two, the OA chondrocytes were transfected with either a pre-mir miRNA precursor for hsa-miR-210 or a pre-mir miRNA negative control precursor (Thermo Fisher Scientific, Inc.) using Lipofectamine RNAiMax (Invitrogen; Thermo Fisher Scientific, Inc.). The final concentration of pre-mir miRNA precursor was $10 \mathrm{nM}$.

HIF-3 $\alpha$ siRNA and negative control siRNA (Santa Cruz Biotechnology, Inc., Santa Cruz, CA, USA) were used to silence the expression of HIF-3 $\alpha$ in the OA chondrocytes. For siRNA transfection, $5 \times 10^{4}$ cells were seeded in each well of 24-well micro-plates, and grown for $24 \mathrm{~h}$ to reach $60-65 \%$ confluence. The cells were then incubated for $15 \mathrm{~min}$ at room temperature with a mixture of siRNA and Lipofectamine 2000 reagent (Invitrogen; Thermo Fisher Scientific, Inc.) in $100 \mu \mathrm{l}$ serum-free OPTI-MEM (Thermo Fisher Scientific, Inc.) according to the manufacturer's protocol. The mRNA and protein levels of HIF-3 $\alpha$ were detected using RT-qPCR and Western blotting, respectively.

Cell proliferation assays. Cell proliferation was determined using XTT assays, which were performed according to the manufacturer's protocol (Roche Diagnostics, Tokyo, Japan). 
A

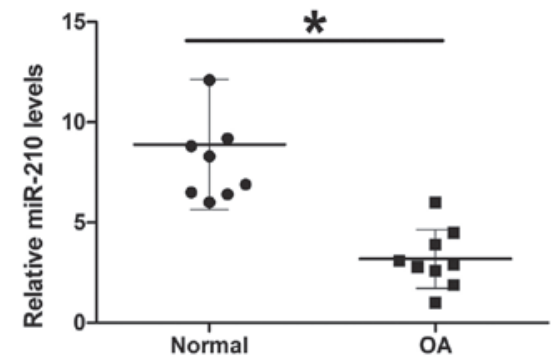

C

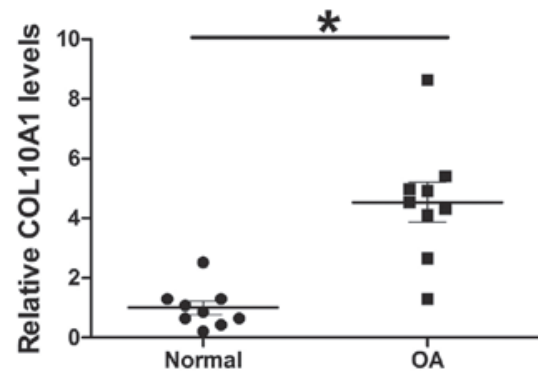

B

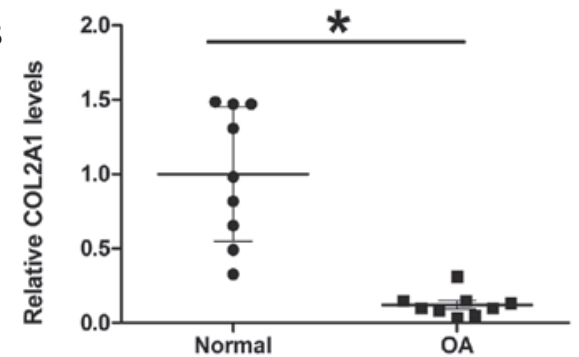

D

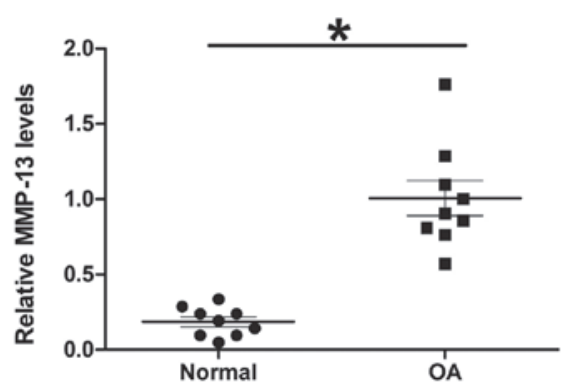

Figure 1. Levels of miR-210, COL2A1, COL10A1 and MMP13 in samples of OA cartilage (n=9) and normal cartilage (n=9). (A) Relative levels of miR-210. (B) Relative mRNA levels of COL2A1. (C) Relative mRNA levels of COL10A1. (D) Relative mRNA levels of MMP-13. The data are presented in a dot plot, with each dot representing the value of one donor. The data are presented as the mean \pm standard deviation. * $\mathrm{P}<0.05$. COL2A1, type II collagen; COL10A1, type X collagen; MMP-13, matrix metalloproteinase 13; miR, microRNA; OA, osteoarthritis.

The cells were seeded into 96 -well plates, at a density of $2 \times 10^{3}$ cells per well, in $100 \mu 1$ culture medium with or without hsa-miR-210, a pre-mir miRNA or HIF-3 $\alpha$ siRNA transfection. The cells were cultured in a $\mathrm{CO}_{2}$ incubator at $37^{\circ} \mathrm{C}$ for $48 \mathrm{~h}$. XTT mixture $(10 \mu \mathrm{l})$ was added to each well and mixed gently, and the cells were incubated for $2 \mathrm{~h}$ at $37^{\circ} \mathrm{C}$ in a $\mathrm{CO}_{2}$ incubator. The absorbance of each sample was measured using a microplate reader (Benchmark Plus; Bio-Rad Laboratories, Inc., Hercules, CA, USA) at a wavelength of $450 \mathrm{~nm}$. Three independent assays were performed.

Western blot analysis. The cells were harvested $72 \mathrm{~h}$ following transfection and were then lysed with RIPA lysis buffer (100 mM NaCl, 50 mM Tris $\mathrm{HCl}$ (pH 7.5), 1\% Triton-X-100, $1 \mathrm{mM}$ EDTA, $10 \mathrm{mM} \beta$-glycerophosphate, $2 \mathrm{mM}$ sodium vanadate). Protein concentration was determined using Bradford Protein Assay kit (Bio-Rad Laboratories, Inc.). A total of $30 \mu \mathrm{g}$ protein was separated by NuPAGE on $4-12 \%$ bis-tris gels (Invitrogen; Thermo Fisher Scientific, Inc.) and transferred onto polyvinylidene fluoride membranes (EMD Millipore, Boston, MA, USA). Nonspecific binding was blocked by incubation with 5\% nonfat milk TBST $(10 \mathrm{mM}$ Tris- $\mathrm{HCl}$ (pH 7.5), $150 \mathrm{mM} \mathrm{NaCl}$, and $0.05 \%$ Tween-20) for $3 \mathrm{~h}$ at room temperature. Subsequent to blocking, primary antibodies were added and incubated overnight at $4{ }^{\circ} \mathrm{C}$. Immunoblotting was performed with anti-HIF-3 $\alpha(1: 1,000$; cat. no. sc-28707; rabbit polyclonal IgG anti-human), anti-Ki67 (1:1,000; cat. no. sc-15402; rabbit polyclonal IgG anti-human) and anti- $\beta$-actin antibodies (1:1,000; cat. no. sc-81760; mouse monoclonal IgM anti-human). All antibodies were sourced from Santa Cruz Biotechnology, Inc. The membranes were incubated with horseradish peroxidase-conjugated secondary antibody (1:5000; cat. no. KC-MM-025; goat monoclonal IgG anti-rabbit; KangChen Bio-tech, Inc., Shanghai, China) for $3 \mathrm{~h}$ at room temperature. Enhanced chemiluminescence (ECL) reagents (Beyotime Institute of Biotechnology, Nantong, China) were used for detection. Specific complexes were visualized using an ECL detection system (GE Healthcare Life Sciences, Little Chalfont, UK).

Dual-luciferase reporter assays. The OA chondrocytes were seeded into 48 -well plates $24 \mathrm{~h}$ prior to transfection $\left(1 \times 10^{4}\right.$ cells/well). HIF-3 $\alpha$ was a target of miR-210 in OA chondrocytes as predicted by TargetScan (http://www.targetscan.org/). The sequences of the HIF-3 $\alpha$ 3'UTR (5'-TCCTCCTACTTCAGCTCCCACAAGTAGCTG GGACTGCAGCTATGTGCCATCATGCCTGGCTGATGTT TATATGTTTTGTAGAGACGAGGTTTCACCATGTTGCCC AGGCTGGTCTTGAACTCCTGAGTTCAAGCGATCCAC CTGCCTTGGCCTCCCAAAGTGCTGGGATTACTGGTAT GAACCACCACGCCCGACAGTAAATATGTTTTGAATG AATAAACTCTCATAAATGA-3') were inserted between the XhoI and PmeI restriction sites in the psiCHECK-2 vector (Promega Corporation, Madison, WI, USA). The cells were first transfected with oligonucleotides (Guangzhou RiboBio Co., Ltd., Guangzhou, China) or miRNA overexpression plasmids (Guangzhou RiboBio Co., Ltd.) using Lipofectamine 2000 (Thermo Fisher Scientific, Inc.), and with reporter vectors on the following day. The activities of firefly and enilla luciferase in the cell lysates were determined using a Dual-Luciferase Reporter Assay system (Promega Corporation). Normalized data were calculated as the ratio of Renilla/firefly luciferase activities.

Statistical analysis. The data are presented as the mean \pm standard deviation. The SPSS 19 software package (IBM SPSS, Armonk, NY, USA) was used for statistical analysis. The data were analyzed using a two-tailed paired t-test. $\mathrm{P}<0.05$ was considered to indicate a statistically significant difference. 

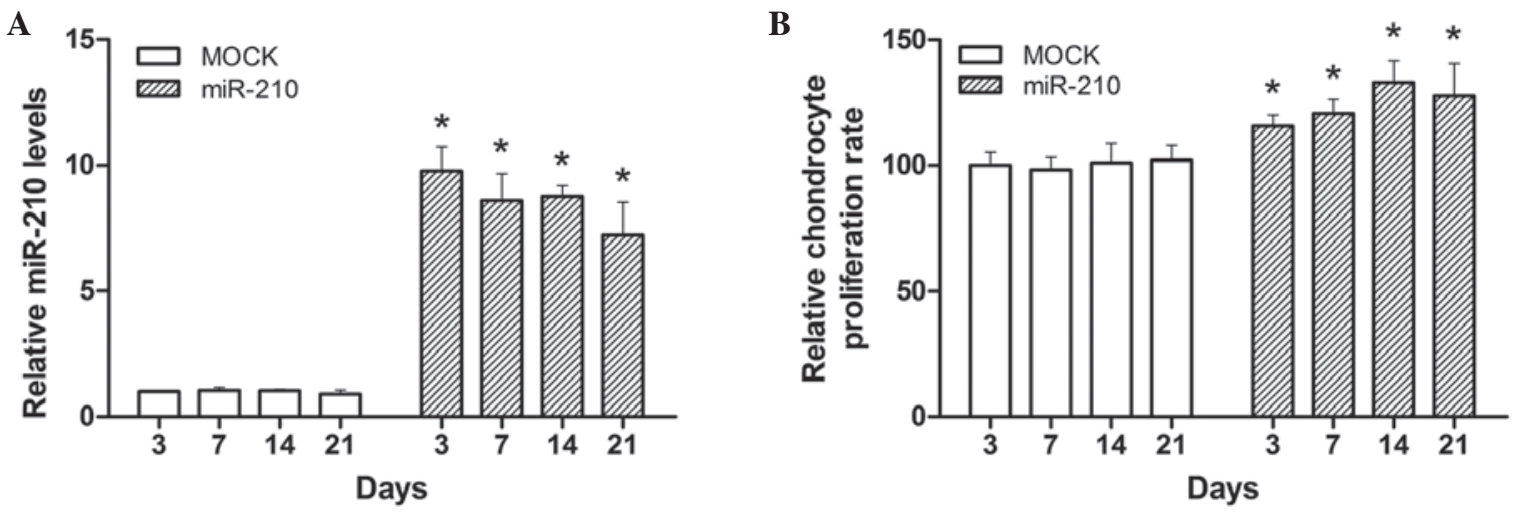

C

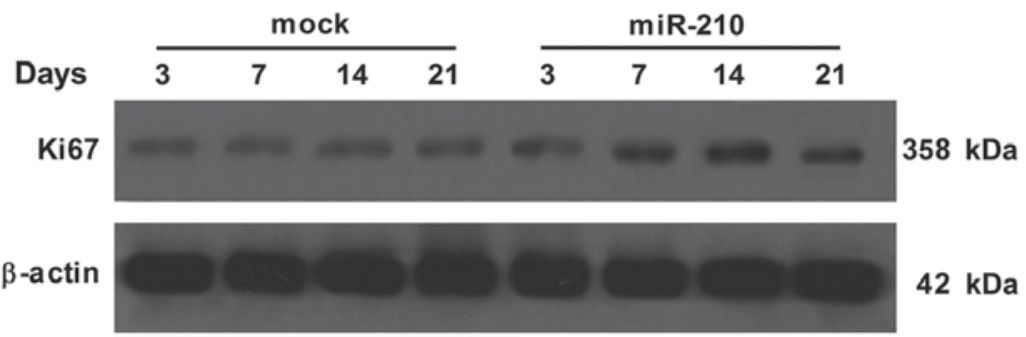

Figure 2. miR-210 increases osteoarthritis chondrocyte proliferation. (A) Expression of miR-210, (B) chondrocyte proliferation and (C) Protein expression of Ki67 were measured 3, 7, 14, and 21 days following transfection, with a pre-mir miRNA precursor for miR-210 or negative control (mock). $\beta$-actin was used as a loading control. The results are presented relative to the mock group and are presented as the mean \pm standard deviation. ${ }^{*} \mathrm{P}<0.05$. miR, microRNA.

A

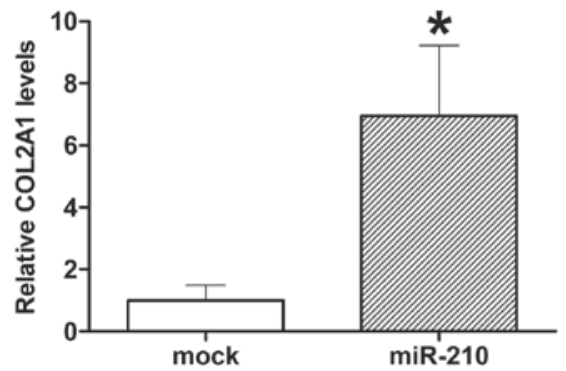

C

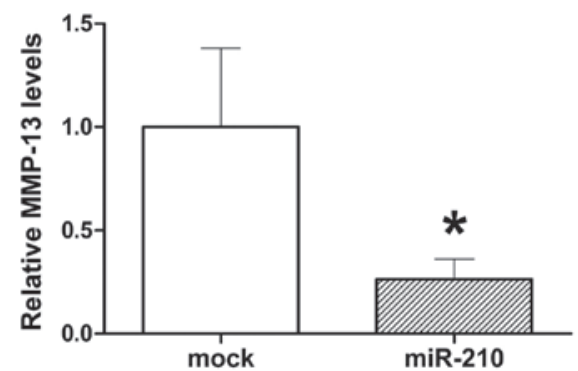

B

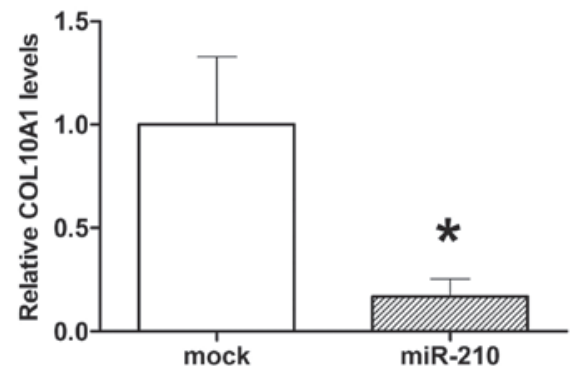

D

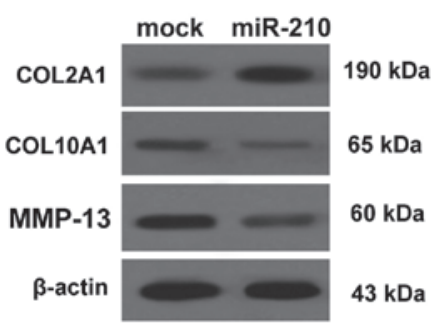

Figure 3. Effect of miR-210 on the mRNA levels of COL2A1, COL10A1 and MMP13 in OA chondrocytes. The mRNA levels of (A) COL2A1, (B) COL10A1 and (C) MMP13, and the (D) protein levels of COL2A1, COL10A1 and MMP13 were measured following transfection with a pre-mir miRNA precursor for miR-210 or negative control (mock). $\beta$-actin was used as an internal control. Data are presented as the mean \pm standard deviation. "P<0.05, vs. mock. COL2A1, type II collagen; COL10A1, type X collagen; MMP13, matrix metalloproteinase 13; miR, microRNA; OA, osteoarthritis.

\section{Results}

Expression of miR-210 is reduced in $O A$ chondrocytes. The expression levels of miR-210 were analyzed and compared using RT-qPCR between the normal and OA cartilage specimens. The expression of miR-210 was decreased by $\sim 10$-fold in the OA cartilage, compared with normal cartilage (Fig. 1A). The mRNA expression levels of COL2A1, COL10A1 and MMP13 were also measured. The expression of COL2A1 was decreased in the OA specimens (Fig. 1B), whereas the expression levels of COL10A1 and MMP13 were increased (Fig. 1C and D).

Overexpression of miR-210 promotes chondrocyte proliferation. To investigate the effects of miR-210 in OA, an miRNA precursor (pre-mir) for miR-210, or a negative control was transfected into the OA chondrocytes. RT-qPCR analysis confirmed that transfection with the pre-mir miR-210 
A

Positions 2099-2120 of HIF-3a 3'UTR

miR-210: $3^{\prime}$ AGUCGG-CGACAGUGUGCGUGUC 5

HIF-3 2 : 3 ' CACGCCGGCAGCCA-ACGCACAG $3^{\prime}$

C

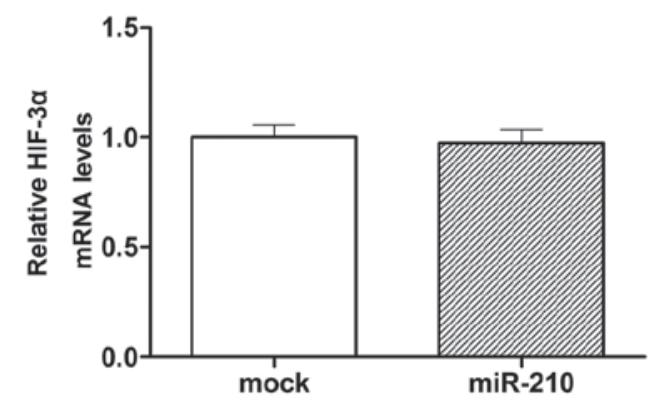

B

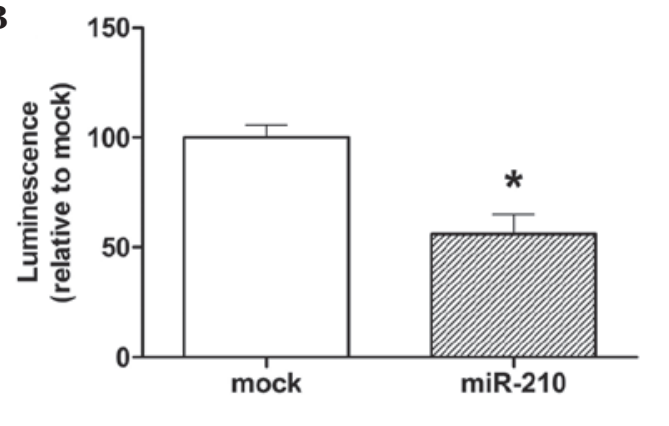

D

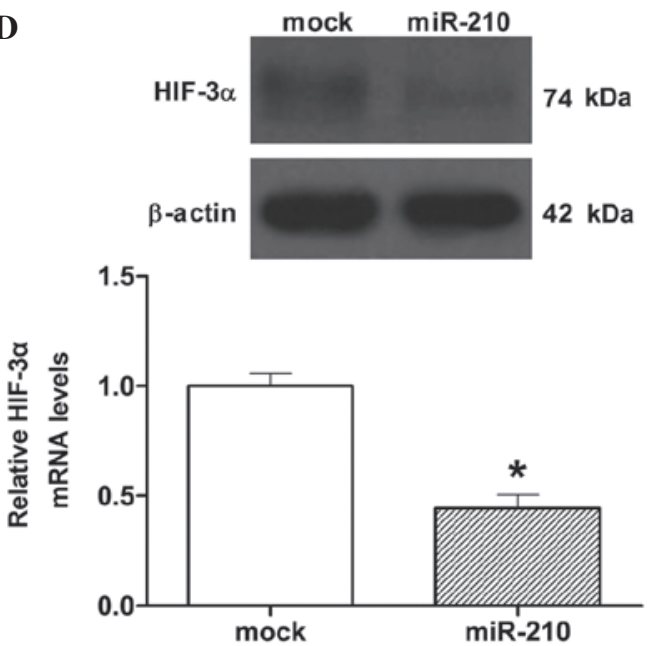

Figure 4. Direct regulation of HIF-3 $\alpha$ by miR-210. The (A) mRNA and (B) protein expression levels of HIF-3 $\alpha$ were evaluated following transfection with a pre-mir miRNA precursor for miR-210 or negative control (mock). $\beta$-actin was used as an internal control. (C) miR-24-1 binding sites in the 3 'UTR of HIF-3 $\alpha$ mRNA. (D) Luciferase reporter assay using a vector encoding putative miR-210 target sites at position 2099-2120. The results are presented relative to the mock control as the mean \pm standard deviation. "P<0.05, vs. mock. miR, microRNA; OA, osteoarthritis; HIF $3 \alpha$, hypoxia-inducible factor-3 $\alpha, 3^{\prime} \mathrm{UTR}$, 3' untranslated region.

A

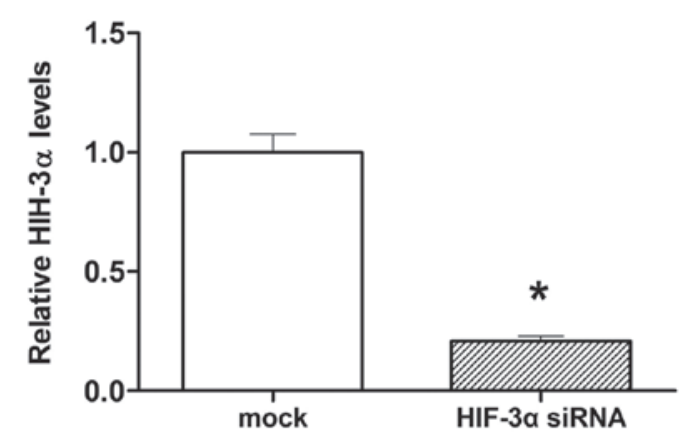

B

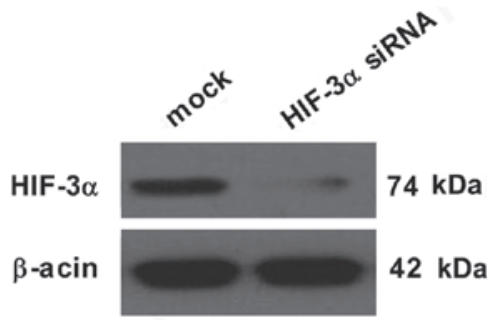

C

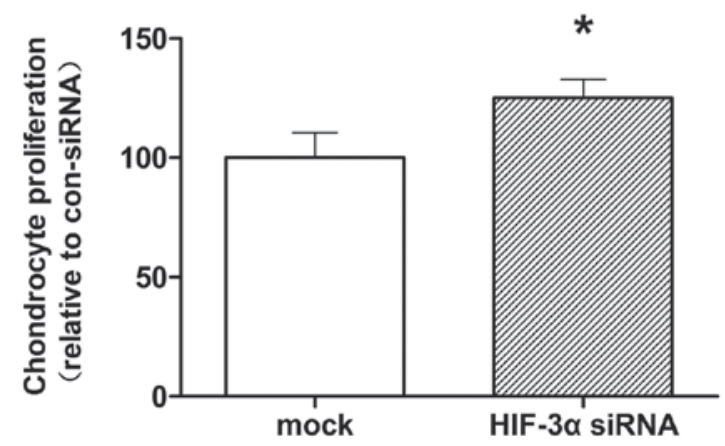

Figure 5. mRNA and protein levels of HIF-3 $\alpha$ following transfection with HIF-3 $\alpha$ siRNA in OA chondrocytes, and the effects of HIF-3 $\alpha$ siRNA on cell proliferation. The (A) mRNA and (B) protein levels of HIF-3 $\alpha$ were measured following transfection with HIF-3 $\alpha$ siRNA or control (con) siRNA (mock). $\beta$-actin was used as an internal control. (C) Chondrocyte proliferation was measured using an XTT assay. The results are presented relative to the mock control as the mean \pm standard deviation. "P<0.05, vs. mock. miR, microRNA; OA, osteoarthritis; HIF $3 \alpha$, hypoxia-inducible factor-3 $\alpha$; siRNA, small interfering RNA. 
A

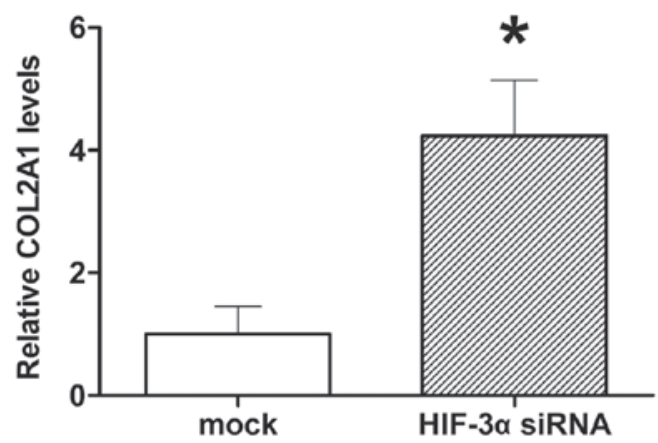

C

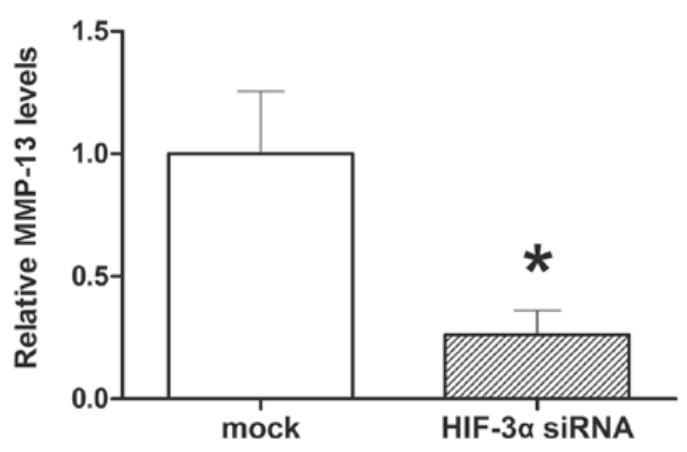

B

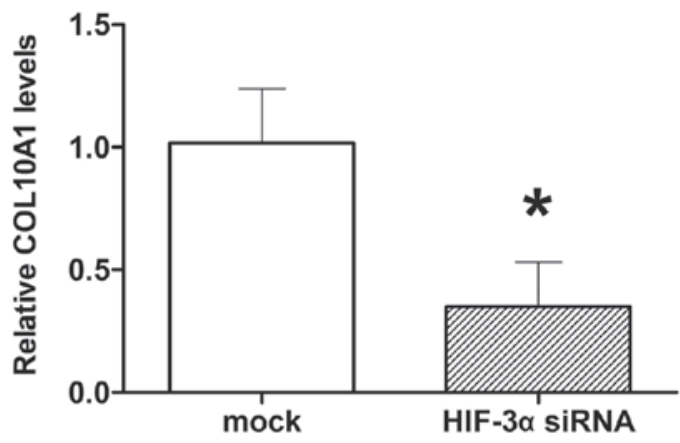

D

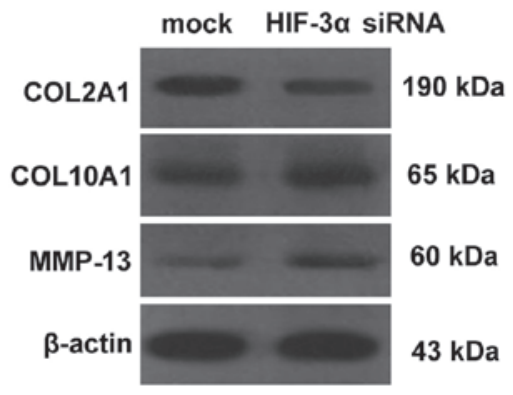

Figure 6. Effects of HIF-3 $\alpha$ siRNA on the levels of COL2A1, COL10A1 and MMP13. mRNA levels of (A) COL2A1, (B) COL10A1 and (C) MMP13 were measured following transfection with HIF-3 $\alpha$ siRNA and control siRNA (mock). $\beta$-actin was used as an internal control. (D) Protein levels of COL2A1, COL10A1 and MMP13 were measured following transfection with HIF-3 $\alpha$ siRNA and control siRNA (mock). $\beta$-actin was used as an internal control. Data are presented as the mean \pm standard deviation. ${ }^{*} \mathrm{P}<0.05$, vs. mock. siRNA, small interfering RNA; COL2A1, type II collagen; COL10A1, type X collagen; MMP13, matrix metalloproteinase 13; miR, microRNA; OA, osteoarthritis; HIF $3 \alpha$, hypoxia-inducible factor-3 $\alpha$.

increased the expression of miR-210, compared with the negative control transfection (Fig. 2B). The expression level of miR-210 was highest 3 days post-transfection, with an almost 5-fold increase, compared with the negative control. Chondrocyte proliferation and the protein expression of $\mathrm{Ki} 67$ were promoted by the overexpression of miR-210 (Fig. 2B and $\mathrm{C}$ ), with the highest proliferation rate, as well as the protein expression levels of Ki67 14 days following transfection.

Overexpression of miR-210 prompts ECM deposition. Cartilage is composed of proteoglycans and type II collagen (18), however, type $\mathrm{X}$ collagen and MMP-13 are involved in cartilage matrix degradation in OA $(19,20)$. To investigate the effect of miR-210 on extracellular matrix deposition, the present study investigated the expression levels of COL2A1 and COL10A1, as well as the levels of MMP13 using RT-qPCR and Western blot analysis. Overexpression of miR-210 resulted in increased mRNA and protein levels of COL2A1, and decreased mRNA and protein levels of COL10A1 and MMP13 in the OA chondrocytes (Fig. 3).

miR-210 directly regulates the expression of $H I F-3 \alpha$. The TargetScan database (http://www.targetscan.org) predicted that there is one binding site for miR-210 in the 3'UTR of HIF-3 $\alpha$ (position 2099-2120; Fig. 4A). To analyze the miRNA-mRNA interactions of the predicted target genes, a luciferase reporter assay was performed in the OA chondrocytes. Vectors containing the partial sequence of the 3'UTR of HIF-3 $\alpha$ mRNA, where the predicted miR-210 target sites were located, were used. Luciferase activity was significantly reduced following the miR-210 transfection, compared with the negative control transfection $(\mathrm{P}<0.05$; Fig. 4B). These data suggested that miR-210 binded directly to the 3'UTR of HIF-3 $\alpha$ mRNA. Subsequently, RT-qPCR and Western blot analyses were performed to confirm the regulation of the expression of HIF-3 $\alpha$ by miR-210 in chondrocytes. miR-210 did not alter the mRNA expression of HIF-3 $\alpha$, however, it significantly suppressed the protein expression of HIF-3 $\alpha$, compared with the negative control transfection $(\mathrm{P}<0.05$; Fig. 4C and D).

Knockdown of HIF-3a promotes OA chondrocyte proliferation. To investigate the role of HIF- $3 \alpha$ in chondrocytes, HIF- $3 \alpha$ knockdown was performed by HIF-3 $\alpha$ siRNA transfection. Initially, the efficiency of HIF- $3 \alpha$ siRNA transfection was evaluated in the chondrocytes. The results of Western blot and RT-qPCR analyses showed that HIF-3 $\alpha$ siRNA effectively suppressed the levels of HIF-3 $\alpha$, compared with the control siRNA in the OA chondrocytes (Fig. 5A and B). The results of the XTT assays revealed that chondrocyte proliferation was promoted by the HIF-3 $\alpha$ siRNA transfectant, compared with the control siRNA, in the chondrocytes (Fig. 5C).

Knockdown of HIF-3a decreases extracellular matrix deposition in OA chondrocytes. HIF-3 $\alpha$ siRNA transfection was performed to investigate the functional role of HIF-3 $\alpha$. The results demonstrated that HIF-3 $\alpha$ siRNA significantly increased the mRNA and protein levels of COL2A1 (Fig. 6A), and decreased the mRNA and protein levels of COL10A1 and MMP13 in the OA chondrocytes (Fig. 6B and C). 


\section{Discussion}

OA is the most common type of joint disease affecting humans (21), and can be triggered and affected by a diverse range of factors, including temperature, age, gender, prior joint injury, obesity, genetic predisposition, and mechanical factors, including malalignment and abnormal joint shape (22-24). A number of studies have focused on OA, however, the mechanism underlying the onset and progression of OA remain to be fully elucidated (25).

Cartilage provides a smooth surface, which allows for an efficient gliding motion during joint movement in normal joints (22). Degradation of articular cartilage is one of the key pathological changes in OA, which is triggered by gradual loss of the ECM, composed predominantly of type II collagen and proteoglycans (26). Chondrocytes in the articular cartilage synthesize several cartilage proteins, which are responsible for construction of the ECM network (25). Transcription profiling has demonstrated that OA chondrocytes possess altered gene expression (27). In the present study, the mRNA levels of COL2A1 in OA chondrocytes were significantly lower than those in normal cartilage, whereas the mRNA levels of COL10A1 and MMP13 were significantly higher. These results reflect reduced cartilage protein expression and the potentiation of cartilage degradation in OA.

Evidence has shown that miRNAs are important in cartilage development and structure in mice (28). For example, miR-27a and $\mathrm{miR}-27 \mathrm{~b}$ have been shown to regulate the expression of MMP-13 in human OA chondrocytes $(29,30)$, and miR-34 $\alpha$ has been reported to modulate chondrocyte apoptosis (31). However, whether miR-210 is involved in the progression of OA remains to be fully elucidated. In the present study, the expression of miR-210 was found to be lower in OA cartilage, compared with healthy cartilage, suggesting that miR-210 may be involved in the pathological process of OA. To investigate this possibility, the expression of miR-210 in OA chondrocytes was upregulated via miR-210 transfection, which was observed to increase OA chondrocyte proliferation, which was accompanied by augmented expression of Ki67. In addition, miR-210 transfection in OA chondrocytes increased the expression of COL2A1, and decreased the expression levels of COL10A1 and MMP13. These results provided evidence supporting miR-210 as a positive regulator of chondrocyte proliferation and ECM deposition in the pathogenesis of OA.

To further understand the mechanism of miR-210 in OA, the present study identified the target of miR-210 using TargetScan. It was predicted that HIF-3 $\alpha$ was a target of miR-210 in OA chondrocytes, and the direct binding of hsa-miR-210 to the HIF-3 $\alpha$ 3'UTR was examined using luciferase reporter assays. HIF- $3 \alpha$ is one isoform of the HIF- $\alpha$ subunit, of which the remaining two subunits are HIF-1 $\alpha$ and HIF-2 $\alpha$, respectively (32). HIF-I modulates adaptive cellular responses in hypoxic environments, and modulates the expression of numerous genes involved in metabolism, cell survival and apoptosis, in an oxygen-dependent manner (33). As cartilage develops in a hypoxic environment, it has been suggested that HIF is involved in the development of chondrocytes (34). Previous evidence has shown that HIF- $\alpha$ is an essential factor for chondrocyte growth arrest and survival (35), and it has been hypothesized that HIF offers potential for cartilage repair (34).
In the present study, miR-210 overexpression suppressed the mRNA and protein expression levels of HIF-3 $\alpha$ in the OA chondrocytes, confirming that HIF-3 $\alpha$ is a target for miR-210 in OA chondrocytes. To further confirm the role of HIF-3 $\alpha$ in the pathogenesis of $\mathrm{OA}$, loss of function experiments using siRNA was performed. The results showed that cell proliferation was inhibited by HIF-3 $\alpha$ knockdown in OA chondrocytes. In addition, the mRNA level of COL2A1 was increased, whereas the mRNA levels of COL10A1 and MMP13 were decreased, following HIF-3 $\alpha$ knockdown.

In conclusion, the present study demonstrated that miR-210 targeted HIF-3 $\alpha$, and thereby enhanced the proliferation of chondrocytes, stimulated the expression of COL2A1 and reduced the expression levels of COL10A1 and MMP13. These effects may render miR-210 a potential target for cartilage repair or OA therapy.

\section{References}

1. Goldring MB: Update on the biology of the chondrocyte and new approaches to treating cartilage diseases. Best Pract Res Clin Rheumatol 20: 1003-1025, 2006.

2. Guo FJ, Xiong Z, Lu X, Ye M, Han X and Jiang R: ATF6 upregulates XBP1S and inhibits ER stress-mediated apoptosis in osteoarthritis cartilage. Cell Signal 26: 332-342, 2014.

3. Goldring MB and Goldring SR: Osteoarthritis. J Cell Physiol 213: 626-634, 2007.

4. Swingler TE, Wheeler G, Carmont V, Elliott HR, Barter MJ, Abu-Elmagd M, Donell ST, Boot-Handford RP, Hajihosseini MK, Münsterberg A, et al: The expression and function of microRNAs in chondrogenesis and osteoarthritis. Arthritis Rheum 64: 1909-1919, 2012.

5. Bartel DP: MicroRNAs: Target recognition and regulatory functions. Cell 136: 215-233, 2009.

6. Filipowicz W, Bhattacharyya SN and Sonenberg N: Mechanisms of post-transcriptional regulation by microRNAs: Are the answers in sight? Nat Rev Genet 9: 102-114, 2008.

7. Wu C, Tian B, Qu X, Liu F, Tang T, Qin A, Zhu Z and Dai K: MicroRNAs play a role in chondrogenesis and osteoarthritis (Review). Int J Mol Med 34: 13-23, 2014.

8. Zhang Y, Jia J, Yang S, Liu X, Ye S and Tian H: MicroRNA-21 controls the development of osteoarthritis by targeting GDF-5 in chondrocytes. Exp Mol Med 46: e79, 2014.

9. Li X, Kroin JS, Kc R, Gibson G, Chen D, Corbett GT, Pahan K, Fayyaz S, Kim JS, van Wijnen AJ, et al: Altered Spinal microRNA-146a and the microRNA-183 cluster contribute to osteoarthritic pain in knee joints. J Bone and Miner Res 28: 2512-2522, 2013

10. Song J, Kim D, Lee CH, Lee MS, Chun $\mathrm{CH}$ and Jin EJ: MicroRNA-488 regulates zinc transporter SLC39A8/ZIP8 during pathogenesis of osteoarthritis. J Biomed Sci 20: 31, 2013.

11. Vonk LA, Kragten AH, Dhert WJ, Saris DB and Creemers LB: Overexpression of hsa-miR-148a promotes cartilage production and inhibits cartilage degradation by osteoarthritic chondrocytes. Osteoarthritis Cartilage 22: 145-153, 2014.

12. Park SJ, Cheon EJ, Lee MH and Kim HA: MicroRNA-127-5p regulates matrix metalloproteinase 13 expression and interleukin-1 $\beta$-induced catabolic effects in human chondrocytes. Arthritis Rheum 65: 3141-3152, 2013.

13. Devlin C, Greco S, Martelli F and Ivan M: miR-210: More than a silent player in hypoxia. IUBMB Life 63: 94-100, 2011.

14. Bodempudi V, Hergert P, Smith K, Xia H, Herrera J, Peterson M, Khalil W, Kahm J, Bitterman PB and Henke CA: miR-210 promotes IPF fibroblast proliferation in response to hypoxia. Am J Physiol Lung Cell Mol Physiol 307: L283-L294, 2014.

15. Li L, Huang K, You Y, Fu X, Hu L, Song L and Meng Y: Hypoxia-induced miR-210 in epithelial ovarian cancer enhances cancer cell viability via promoting proliferation and inhibiting apoptosis. Int J Oncol 44: 2111-2120, 2014.

16. Chang Z, Huo L, Wu Y and Zhang P: HIF-1 $\alpha$ had pivotal effects on downregulation of miR-210 decreasing viability and inducing apoptosis in hypoxic chondrocytes. Scientific WorldJournal 2014: 876363, 2014. 
17. Pfaffl MW: A new mathematical model for relative quantification in real-time RT-PCR. Nucleic Acids Res 29: e45, 2001.

18. Burrage PS, Mix KS and Brinckerhoff CE: Matrix metalloproteinases: Role in arthritis. Front Biosci 11: 529-543, 2005

19. Mackie EJ, Tatarczuch L and Mirams M: The skeleton: A multi-functional complex organ. The growth plate chondrocyte and endochondral ossification. J Endocrinol 211: 109-121, 2011

20. Loeser RF: Osteoarthritis year in review 2013: Biology. Osteoarthritis Cartilage 21: 1436-1442, 2013.

21. Lawrence RC, Felson DT, Helmick CG, Arnold LM, Choi H, Deyo RA, Gabriel S, Hirsch R, Hochberg MC, Hunder GG, et al: Estimates of the prevalence of arthritis and other rheumatic conditions in the United States: Part II. Arthritis Rheum 58: 26-35, 2008.

22. Loeser RF, Goldring SR, Scanzello CR and Goldring MB: Osteoarthritis: A disease of the joint as an organ. Arthritis Rheum 64: 1697-1707, 2012.

23. Felson DT, Lawrence RC, Dieppe PA, Hirsch R, Helmick CG, Jordan JM, Kington RS, Lane NE, Nevitt MC, Zhang Y, et al: Osteoarthritis: New insights. Part 1: The disease and its risk factors. Ann Intern Med 133: 635-646, 2000.

24. Blagojevic M, Jinks C, Jeffery A and Jordan K: Risk factors for onset of osteoarthritis of the knee in older adults: A systematic review and meta-analysis. Osteoarthritis Cartilage 18: 24-33, 2010

25. Staal B, Williams BO, Beier F, Vande Woude GF and Zhang YW: Cartilage-specific deletion of Mig-6 results in osteoarthritis-like disorder with excessive articular chondrocyte proliferation. Proc Natl Acad Sci USA 111: 2590-2595, 2014.

26. Tchetina EV: Developmental mechanisms in articular cartilage degradation in osteoarthritis. Arthritis 2011: 683970, 2011.

27. Aigner T, Fundel K, Saas J, Gebhard PM, Haag J, Weiss T, Zien A, Obermayr F, Zimmer R and Bartnik E: Large-scale gene expression profiling reveals major pathogenetic pathways of cartilage degeneration in osteoarthritis. Arthritis Rheum 54: 3533-3544, 2006.
28. Kobayashi T, Lu J, Cobb BS, Rodda SJ, McMahon AP, Schipani E, Merkenschlager $M$ and Kronenberg HM: Dicer-dependent pathways regulate chondrocyte proliferation and differentiation. Proc Natl Acad Sci USA 105: 1949-1954, 2008.

29. Tardif G, Hum D, Pelletier JP, Duval N and Martel-Pelletier J: Regulation of the IGFBP-5 and MMP-13 genes by the microRNAs miR-140 and miR-27a in human osteoarthritic chondrocytes. BMC Musculoskelet Disord 10: 148, 2009.

30. Akhtar N, Rasheed Z, Ramamurthy S, Anbazhagan AN, Voss FR and Haqqi TM: MicroRNA-27b regulates the expression of matrix metalloproteinase 13 in human osteoarthritis chondrocytes. Arthritis Rheum 62: 1361-1371, 2010.

31. Abouheif MM, Nakasa T, Shibuya H, Niimoto T, Kongcharoensombat W and Ochi M: Silencing microRNA-34a inhibits chondrocyte apoptosis in a rat osteoarthritis model in vitro. Rheumatology (Oxford) 49: 2054-2060, 2010.

32. Heikkilä M, Pasanen A, Kivirikko KI and Myllyharju J: Roles of the human hypoxia-inducible factor (HIF)-3 $\alpha$ variants in the hypoxia response. Cell Mol Life Sci 68: 3885-3901, 2011.

33. Ando H, Natsume A, Iwami K, Ohka F, Kuchimaru T, Kizaka-Kondoh S, Ito K, Saito K, Sugita S, Hoshino T and Wakabayashi T: A hypoxia-inducible factor (HIF)-3 $\alpha$ splicing variant, HIF-3a4 impairs angiogenesis in hypervascular malignant meningiomas with epigenetically silenced HIF-3 $\alpha 4$. Biochem Biophys Res Commun 433: 139-144, 2013.

34. Murphy CL, Thoms BL, Vaghjiani RJ and Lafont JE: Hypoxia. HIF-mediated articular chondrocyte function: Prospects for cartilage repair. Arthritis Res Ther 11: 213, 2009.

35. Schipani E, Ryan HE, Didrickson S, Kobayashi T, Knight M and Johnson RS: Hypoxia in cartilage: HIF-1alpha is essential for chondrocyte growth arrest and survival. Genes Dev 15: 2865-2876, 2001. 